\title{
On the Diversity of Malaria Parasites in African Apes and the Origin of Plasmodium falciparum from Bonobos
}

\author{
Sabrina Krief ${ }^{19}$, Ananias A. Escalante ${ }^{29}$, M. Andreina Pacheco ${ }^{2}$, Lawrence Mugisha ${ }^{3}$, Claudine André ${ }^{4}$, \\ Michel Halbwax ${ }^{5}$, Anne Fischer ${ }^{5 x}$, Jean-Michel Krief ${ }^{6}$, John M. Kasenene ${ }^{7}$, Mike Crandfield ${ }^{8}$, Omar E. \\ Cornejo ${ }^{9}$, Jean-Marc Chavatte ${ }^{10}$, Clara Lin ${ }^{11}$, Franck Letourneur ${ }^{12}$, Anne Charlotte Grüner ${ }^{11,12}$, Thomas F. \\ McCutchan $^{13}$, Laurent Rénia ${ }^{11,12}$, Georges Snounou ${ }^{10,11,14,15,16 *}$
}

1 UMR 7206-USM 104, Eco-Anthropologie et Ethnobiologie, Muséum National d'Histoire Naturelle, Paris, France, 2 School of Life Sciences, Arizona State University, Tempe, Arizona, United States of America, 3 Chimpanzee Sanctuary \& Wildlife Conservation Trust (CSWCT), Entebbe, Uganda, $\mathbf{4}$ Lola Ya Bonobo Bonobo Sanctuary, “Petites Chutes de la Lukaya", Kimwenza-Mont Ngafula, Kinshasa, Democratic Republic of Congo, 5 Max-Planck Institute for Evolutionary Anthropology, Leipzig, Germany, 6 Projet pour la Conservation des Grands Singes, Paris, France, 7 Department of Botany, Makerere University, Kampala, Uganda; Makerere University Biological Field Station, Fort Portal, Uganda, 8 Research and Conservation Program, The Maryland Zoo in Baltimore, Baltimore, Maryland, United States of America, 9 Emory University, Program in Population Biology, Ecology, and Evolution, Atlanta, Georgia, United States of America, 10 USM0307, Parasitologie Comparée et Modèles Expérimentaux, Muséum National d'Histoire Naturelle, Paris, France, 11 Laboratory of Malaria Immunobiology, Singapore Immunology Network, Agency for Science Technology and Research (A*STAR), Biopolis, Singapore, 12 Institut Cochin, Université Paris Descartes, CNRS (UMR 8104), Paris, France; INSERM U567, Paris, France, 13 Laboratory of Malaria and Vector Research, National Institute of Allergy and Infectious Diseases, National Institute of Health, Bethesda, Maryland, United States of America, 14 INSERM UMR S 945 , Paris, France, 15 Université Pierre \& Marie Curie, Faculté de Médecine Pitié-Salpêtrière, Paris, France, 16 Department of Microbiology, National University of Singapore, Singapore

\begin{abstract}
The origin of Plasmodium falciparum, the etiological agent of the most dangerous forms of human malaria, remains controversial. Although investigations of homologous parasites in African Apes are crucial to resolve this issue, studies have been restricted to a chimpanzee parasite related to $P$. falciparum, $P$. reichenowi, for which a single isolate was available until very recently. Using PCR amplification, we detected Plasmodium parasites in blood samples from 18 of 91 individuals of the genus Pan, including six chimpanzees (three Pan troglodytes troglodytes, three Pan $t$. schweinfurthii) and twelve bonobos (Pan paniscus). We obtained sequences of the parasites' mitochondrial genomes and/or from two nuclear genes from 14 samples. In addition to $P$. reichenowi, three other hitherto unknown lineages were found in the chimpanzees. One is related to $P$. vivax and two to $P$. falciparum that are likely to belong to distinct species. In the bonobos we found $P$. falciparum parasites whose mitochondrial genomes indicated that they were distinct from those present in humans, and another parasite lineage related to $P$. malariae. Phylogenetic analyses based on this diverse set of Plasmodium parasites in African Apes shed new light on the evolutionary history of $P$. falciparum. The data suggested that $P$. falciparum did not originate from $P$. reichenowi of chimpanzees (Pan troglodytes), but rather evolved in bonobos (Pan paniscus), from which it subsequently colonized humans by a host-switch. Finally, our data and that of others indicated that chimpanzees and bonobos maintain malaria parasites, to which humans are susceptible, a factor of some relevance to the renewed efforts to eradicate malaria.
\end{abstract}

Citation: Krief S, Escalante AA, Pacheco MA, Mugisha L, André C, et al. (2010) On the Diversity of Malaria Parasites in African Apes and the Origin of Plasmodium falciparum from Bonobos. PLoS Pathog 6(2): e1000765. doi:10.1371/journal.ppat.1000765

Editor: L. David Sibley, Washington University School of Medicine, United States of America

Received September 4, 2009; Accepted January 13, 2010; Published February 12, 2010

This is an open-access article distributed under the terms of the Creative Commons Public Domain declaration which stipulates that, once placed in the public domain, this work may be freely reproduced, distributed, transmitted, modified, built upon, or otherwise used by anyone for any lawful purpose.

Funding: This study was financially supported by the Museum National d'Histoire Naturelle (Paris, France), the Fyssen Foundation and a Leakey grant to SK. AAE was supported by an R01 grant (GM080586) from the US National Institutes of Health. The US NIH intramural program supported TFM. LR, ACG, CL and GS were supported by INSERM and the Agency for Science, Technology and Research (A*STAR), Singapore. The funders had no role in study design, data collection and analysis, decision to publish, or preparation of the manuscript.

Competing Interests: The authors have declared that no competing interests exist.

*E-mail: Ananias.Escalante@asu.edu (AAE); georges.snounou@upmc.fr or gsnounou@gmail.com (GS)

9 These authors contributed equally to this work.

a Current address: African Insect Science for Food and Health, Nairobi, Kenya

\section{Introduction}

Malaria infections have influenced the development of human civilizations, and have shaped the genetic make-up of current human populations. There are four globally distributed Plasmodium protozoan parasites that are responsible for malaria in humans (P. falciparum, $P$. vivax, $P$. malariae and $P$. ovale). Molecular phylogenetic analyses have demonstrated that these four parasites are not monophyletic [1,2], indicating that they independently colonised hominids [3-6]. The timing of their appearance in Homo sapiens, however, remains unresolved. This is of some importance to current efforts to control malaria, because it will affect how observed patterns of genetic diversity in the parasite populations are interpreted. For example, several evolutionary genetic approaches rely on reliable phylogenetic information to detect putative adaptive genetic variation, thereby identifying genes that might be involved in pathogenesis or in the evasion of host immune responses. Addressing these issues is a 


\section{Author Summary}

Chimpanzees and gorillas are known to have malaria parasites (genus Plasmodium) similar to those that infect humans. It is likely that detailed molecular studies of these parasites will help understand important aspects of the malaria disease and of immune defences in humans, and could then guide the development of novel control measures. However, few studies of parasites in African Apes have been conducted to date. Here we present the results of a survey of malaria parasites in chimpanzees and bonobos, our closest relatives. In chimpanzees, we identified two new parasite species closely related to $P$. falciparum, the most dangerous of the parasites in humans. We also found that bonobos harbour malaria parasites including $P$. falciparum. Phylogenetic analyses of these parasites strongly suggested that $P$. falciparum evolved in bonobos, and that it was introduced into humans from bonobos at a later date. Overall, our findings have substantially altered our perception of the origin of malaria parasites in humans.

matter of great importance for $P$. falciparum, the parasite responsible for a substantial proportion of the global malaria mortality and morbidity [7]. It is now generally accepted that $P$. falciparum underwent a population expansion in humans $[4,6,8-$ 11], though how, when and from where humans first acquired $P$. falciparum, is less well established. Suggestions of a host-switch from a chimpanzee parasite received recent support, albeit without resolving the likelihood or timing of this event $[4,10,12]$.

The accuracy and robustness of conclusions derived from comparative analyses (phylogenetic or genomics) will be significantly enhanced if data from all of the evolutionary close parasites were to be included. In the context of parasites of humans, this data would be best obtained from Plasmodium species that infect our nearest relatives, the African Apes, because two of the parasite species, $P$. reichenowi and $P$. rodhaini, that have been reported in Pan and Gorilla are morphologically very similar to $P$. falciparum and $P$. malariae respectively, while the third, $P$. schwetzi, corresponds to $P$. vivax or $P$. ovale $[13,14]$. Studies of the malaria parasites of African Apes have been limited to few observations made mainly in the $1920 \mathrm{~s}-1950 \mathrm{~s}$, and very little is known of their natural history. Nonetheless, it is known that chimpanzees are susceptible to infection by the four parasite species of humans, while humans have been infected with $P$. rodhaini and $P$. schwetzi $[13,14]$. The origin and evolutionary history of the malaria parasites in chimpanzees and gorillas are speculative $[13,14]$ mainly because the molecular data has been restricted to sequences derived from a single $P$. reichenowi isolate $[3,4,8,15,16]$ until very recently [12]. In another recent publication, a novel parasite lineage close to, but distinct from, $P$. reichenow $i$ was reported from chimpanzees sampled in Gabon [17]. This raises the important question as to whether Plasmodium species close to P. falciparum, other than the two described so far, occur in non-human higher primates.

We were afforded a rare opportunity to analyze blood samples collected independently from chimpanzees and bonobos for the presence of Plasmodium parasites. Such a collection of fresh isolates would provide sequence data for improved phylogenetic analyses. Here we report on our findings of a genetically diverse set of Plasmodium parasites found in some of the samples we analyzed, and we discuss the insights they have provided into the origin of the Plasmodium falciparum.

\section{Results}

Blood samples were obtained from 49 chimpanzees, Pan troglodytes, in Uganda and the Democratic Republic of the Congo (DRC), and from 42 bonobos, Pan paniscus, in the DRG. Blood smears were not made available, so the presence and level of Plasmodium parasites were assessed solely by a highly sensitive PCR assay, where a small fragment of the small subunit ribosomal RNA (ssrRNA) genes is amplified using oligonucleotides that target sequences conserved in all known Plasmodium species [18]. Parasites were detected in 18 animals: $3 / 3$ Pan $t$. schweinfurthii living wild in Kibale National Park in Uganda, and in 3/8 Pan t. troglodytes and 12/42 Pan paniscus cared for in sanctuaries in the DRC. Parasitaemias were quite low $(<100$ parasites per $\mu$ of blood), consistent with previous observations of naturally infected apes $[13,14]$.

We opted to conduct our analyses on the DNA purified directly from the blood samples, because whole genome amplification could lead to artefactual recombination between DNA molecules from different strains or species of parasites, should any be present in a given sample. Given the low parasite densities in the samples and the limited blood volumes available, efforts were directed at characterizing a small number of genes that have been used in recent phylogenetic analyses. Specifically, we targeted the mitochondrial genome using oligonucleotide primers that correspond to sequences conserved in Plasmodium. Since we were particularly interested in lineages related to $P$. falciparum, we used oligonucleotides based on sequences from $P$. falciparum to target two nuclear genes: dihydrofolate reductase-thymidylate synthase $(d h f r-t s)$, and the gene encoding the merozoite surface protein 2 (msp2) because this gene is not known to have orthologues outside $P$. falciparum and P. reichenowi [15]. We specifically targeted the block 3 of $m s p 2$, because we hypothesized that the extensive polymorphisms observed for this region in $P$. falciparum might also occur in orthologous genes that could be present in closely related species, and this could provide an indication of genetic diversity in these parasites.

In order to minimize artefacts, nearly all the sequences obtained for the $d h f r$-ts and the $m s p 2$ block 3 fragments were derived from duplicate amplifications. The mitochondrial genome sequences were also derived from duplicate amplification of a single $5800 \mathrm{bp}$ fragment, which spans nearly the complete mitochondrial genome of ca. $6 \mathrm{~kb}$. This avoided any ambiguities in a final assembly of overlapping fragments that might arise from a sample with multiple parasite lineages. Indeed, it was not possible to combine the $d h f r-t s, m s p 2$ and mitochondrial data sets in the subsequent phylogenetic analyses, because mixed infections were common in our samples. Finally, we are confident that cross-contamination during amplification was highly unlikely because similar sequences for the different chimpanzee parasite lineages were derived from samples collected independently in Uganda or the DRC, and then processed in France or in the USA, respectively. Successful amplification was not achieved for all the genes targeted from each sample, and this was particularly noted for the samples from the bonobos. Nonetheless, the sequence data obtained revealed a rich diversity of species and strains (Table S1), in particular for the individual samples collected from the two Pan troglodytes subspecies.

Sixteen near-complete mitochondrial genomes that coalesce in six distinct lineages were obtained from 12 of the 18 samples positive for Plasmodium (Fig. 1). All our phylogenetic analyses lead to identical topologies (see Methods), and only the Bayesian phylogenetic tree is reported (Fig. 1). Two lineages shared a recent common ancestor either with the P. malariae clade (two bonobos) or with the $P$. vivax clade (one chimpanzee from Uganda and one 


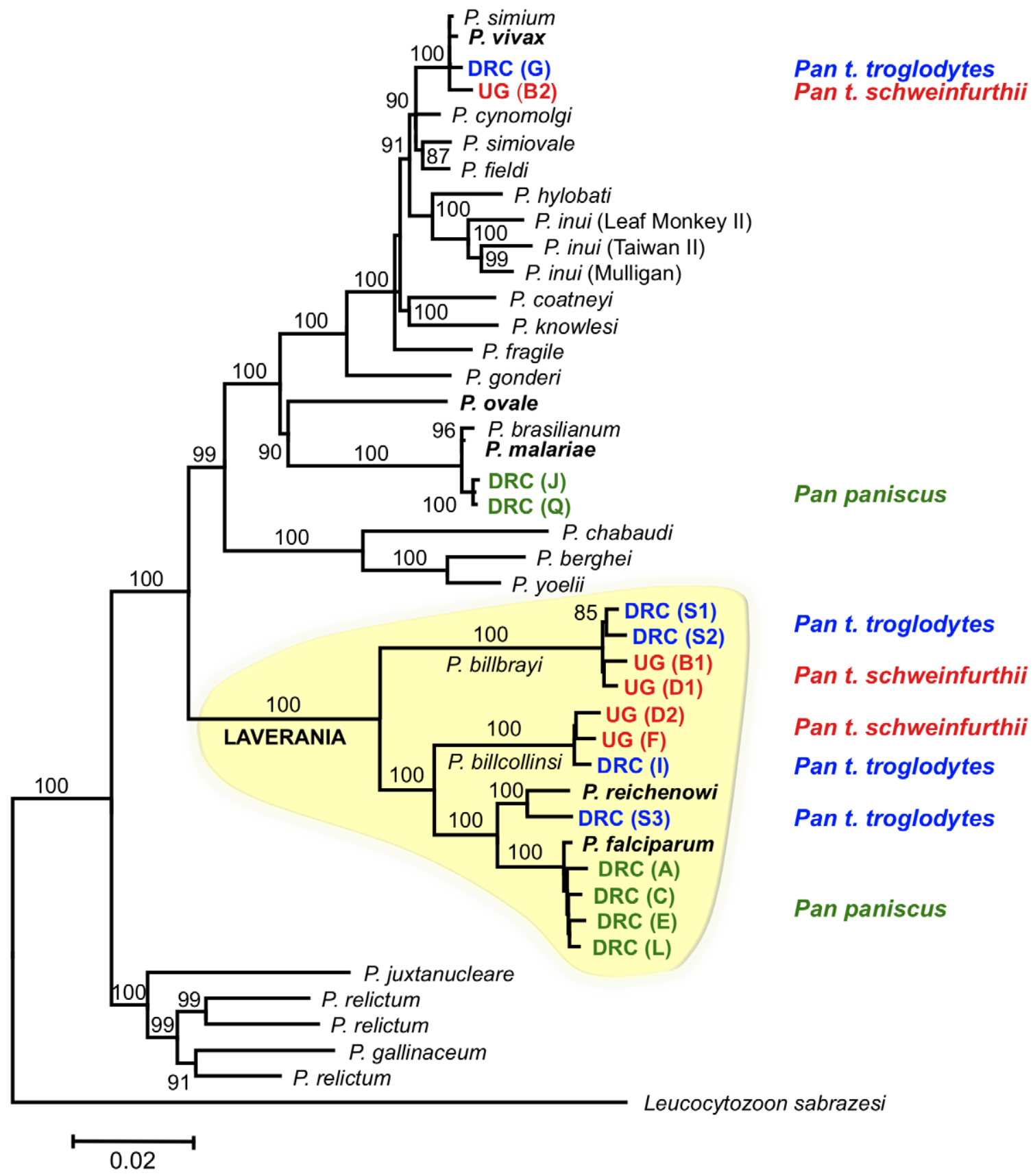

Figure 1. Phylogenetic tree of Plasmodium based on mitochondrial genomes. In the Bayesian phylogenetic tree presented, the values above branches are posterior probabilities expressed as percentages. Maximum likelihood and Bayesian methods lead to identical phylogenies. The names of the species that normally infect humans or chimpanzees are presented in bold. The sequence of the mitochondrial genomes derived from the Ape samples were named (also presented in bold) according to the country in which an Ape was sampled (DRC or UG, which stand for the Democratic Republic of Congo and Uganda, respectively), followed in parentheses by a single letter that indicates the particular Ape from which the sequence was obtained, and a number when two or more distinct sequence were obtained from the sample. Theses names were colour-coded according to the host species, indicated on the right, from which the sequences were derived (Pan t. troglodytes in blue; Pan t. schweinfurthii in red, and Pan paniscus in green). The Laverania clade is highlighted in yellow, and the branches carrying the sequences from the two novel lineages are labelled as the new species to which we propose they belong. The accession numbers of the sequences derived from the parasites found in chimpanzees and bonobos are provided in Table S1, and those of the other species are provided in the Methods. doi:10.1371/journal.ppat.1000765.g001

from the DRC). Another lineage, found in the bonobo samples, clustered with P. falciparum. One lineage from a DRC chimpanzee shared a recent common ancestor with $P$. reichenowi, while the two remaining lineages found in chimpanzees sampled in Uganda and the DRC, were novel and formed a monophyletic group with those of $P$. falciparum and $P$. reichenowi. For the sake of clarity, we have used the name Laverania to refer to this monophyletic clade, a generic name previously proposed to distinguish $P$. falciparum and $P$. reichenowi from the other malaria parasite species (International Commission on Zoological Nomenclature, Opinion 283). We 
hypothesized that the two new lineages in the Laverania clade correspond to two distinct Plasmodium species. This hypothesis was further supported by three other analyses. First, the extent of divergence in the genetic distances between these two novel Laverania lineages, as calculated from the mitochondrial genomes (Table 1), is comparable to that observed between well-established species in the rodent malaria clade, or between $P$. falciparum and $P$. reichenowi. Second, the topology of the phylogenetic tree constructed using $d h f r$-ts sequences from the same isolates reproduces that obtained for the mitochondrial genome (Fig. 2). Indeed, it would appear that an insert coding for eight amino acids is specific to the Laverania lineages ( $P$. falciparum, $P$. reichenowi and the two new lineages), which further supports our conclusion that these lineages form a monophyletic group. Finally, the samples that harboured the two novel lineages and the $P$. reichenowi lineage, yielded $m s p 2$ block 3 sequences that could be grouped into five distinct allelic families, of which one was similar to that previously published for $P$. reichenowi (Fig. 3), while the other four were novel. By a way of comparison, only two allelic families have been identified for the $P$. falciparum msp2 block 3 despite extensive sampling.

Six of the eight bonobos positive for Plasmodium, harboured parasites that yielded sequence data for $d h f r$-ts and/or $m s p 2$. The msp2 and all the dhfr-ts sequences were indistinguishable from known $P$. falciparum sequences. This confirmed that bonobos were infected with P. falciparum, as had been indicated by the sequences of the mitochondrial genomes derived from four of these six bonobos (Fig. 1). Interestingly, we found significant differences in the genetic diversity of the $P$. falciparum mitochondrial lineages derived from bonobos as compared with that previously noted for large set of mitochondrial $P$. falciparum lineages obtained from human isolates collected worldwide [9]. Indeed, the P. falciparum lineages in bonobos $(n=4, \pi=0.0048)$ were ten times more diverse that those found in humans $(n=96, \pi=0.00034)$. Furthermore, there were no fixed differences between the $P$. falciparum from bonobos and those from humans. In other words, the four mitochondrial P. falciparum haplotypes we obtained from the bonobos had each a distinctive set of mutations such that none of these haplotypes were represented in the extensive P. falciparum mitochondrial haplotype database. This is clearly illustrated in the mitochondrial genome haplotype network (Fig. 4). The $P$. falciparum populations from bonobos and from humans, though related, have undergone some level of differentiation. Moreover, the haplotype network indicates that the four haplotypes from the bonobo do not form a monophyletic group, which suggests a scenario where bonobos and humans exchanged parasites in relatively recent times.

\section{Discussion}

The sum of our knowledge on the Plasmodium parasites of African Apes derives from observations, nearly all made before the 1960s, on fewer than 50 naturally infected animals captured primarily in Cameroon, Sierra Leone or the Congo. Given the highly protected status of African Apes, prospects to extend this knowledge are restricted to molecular analyses of blood samples, mainly collected during medical examination of Apes cared for in sanctuaries, or upon recovery from poachers or villagers. The results from three such surveys published this year $[12,17,19]$ have provided new glimpses into the diversity of malaria parasites in chimpanzees, and have allowed testing of hypotheses concerning the evolution of $P$. falciparum $[12,17,19]$. Here we present the outcome of two further independent surveys, one of which is distinguished by the inclusion of samples from bonobos and from wild-living chimpanzees. The molecular data we present demonstrate that the Pan genus naturally harbours a rich Plasmodium fauna, including two novel lineages close to $P$. falciparum, one related to $P$. vivax, and one related to $P$. malariae. Furthermore, it brings to light the presence of a population of $P$. falciparum in bonobos that appears to differ from those in humans. The observations add new perspectives to the evolutionary hypotheses formulated for the Plasmodium parasites of African Great Apes and humans.

From a parasitological point of view, the fact that the three samples collected from Eastern Chimpanzees (Pan t. schweinfurthii) living wild in a community of 44 animals, were all positive and harboured complex mixed strain/species infections (Table S1), suggests that prevalence of infections under natural conditions of transmission is high. This view is supported by our observations of a similar level of parasite diversity in three of the eight Central Chimpanzees (Pan t. troglodytes) that were independently sampled in the DRC (Table S1). It would be interesting to establish whether the other two chimpanzee subspecies, the Western Chimpanzee (Pan $t$. verus) and the Nigeria-Cameroon Chimpanzee (Pan $t$. vellerosus) also harbour the same parasite species. The bonobos cared for in a sanctuary also had high parasite prevalence, with Plasmodium detected via ssrRNA amplification in 12 of the 42 sampled $(28.5 \%)$.

Table 1. Genetic distances between the mitochondrial lineages (ca. $5800 \mathrm{bp}$ ) from selected Plasmodium species.

\begin{tabular}{|c|c|c|c|c|c|c|c|c|c|}
\hline Species & P. ber. & P. yoe. & P. cha. & P. fal. & P. rei. & P. bbr. (S1) & P. bbr. (S2) & P. rei.(S1) & P. bco. \\
\hline P. ber. & - & & & & & & & & \\
\hline P. yoe. & 0.0132 & - & & & & & & & \\
\hline P. cha. & 0.0308 & 0.0286 & - & & & & & & \\
\hline P. fal. & 0.0756 & 0.0747 & 0.0747 & - & & & & & \\
\hline P. rei. & 0.0766 & 0.0761 & 0.0773 & 0.0104 & - & & & & \\
\hline P. bbr. (S1) & 0.0850 & 0.0832 & 0.0839 & 0.0434 & 0.0430 & - & & & \\
\hline P. bbr. (S2) & 0.0860 & 0.0834 & 0.0846 & 0.0441 & 0.0437 & 0.0036 & - & & \\
\hline P. rei.(S3) & 0.0796 & 0.0785 & 0.0785 & 0.0166 & 0.0115 & 0.0390 & 0.0397 & - & \\
\hline P. bco. & 0.0836 & 0.0815 & 0.0820 & 0.0339 & 0.0348 & 0.0390 & 0.0397 & 0.0293 & - \\
\hline
\end{tabular}

Species that infect rodents: $P$. ber $=P$. berghei; $P$. yoe. $=P$. yoelii; $P$. cha $=P$. chabaudi. Species that infect higher primates: $P$. fal $=P$. falciparum; $P$. rei $=P$. reichenowi (S3 in brackets indicated the haplotype DRC (S3) identified in this study). The new species described in this study are: $P$. bbr. = P. billbrayi (haplotype DRC (S1), DRC (S2)); P. bcol = P. billcollinsi (haplotype DRC (I)).

doi:10.1371/journal.ppat.1000765.t001 

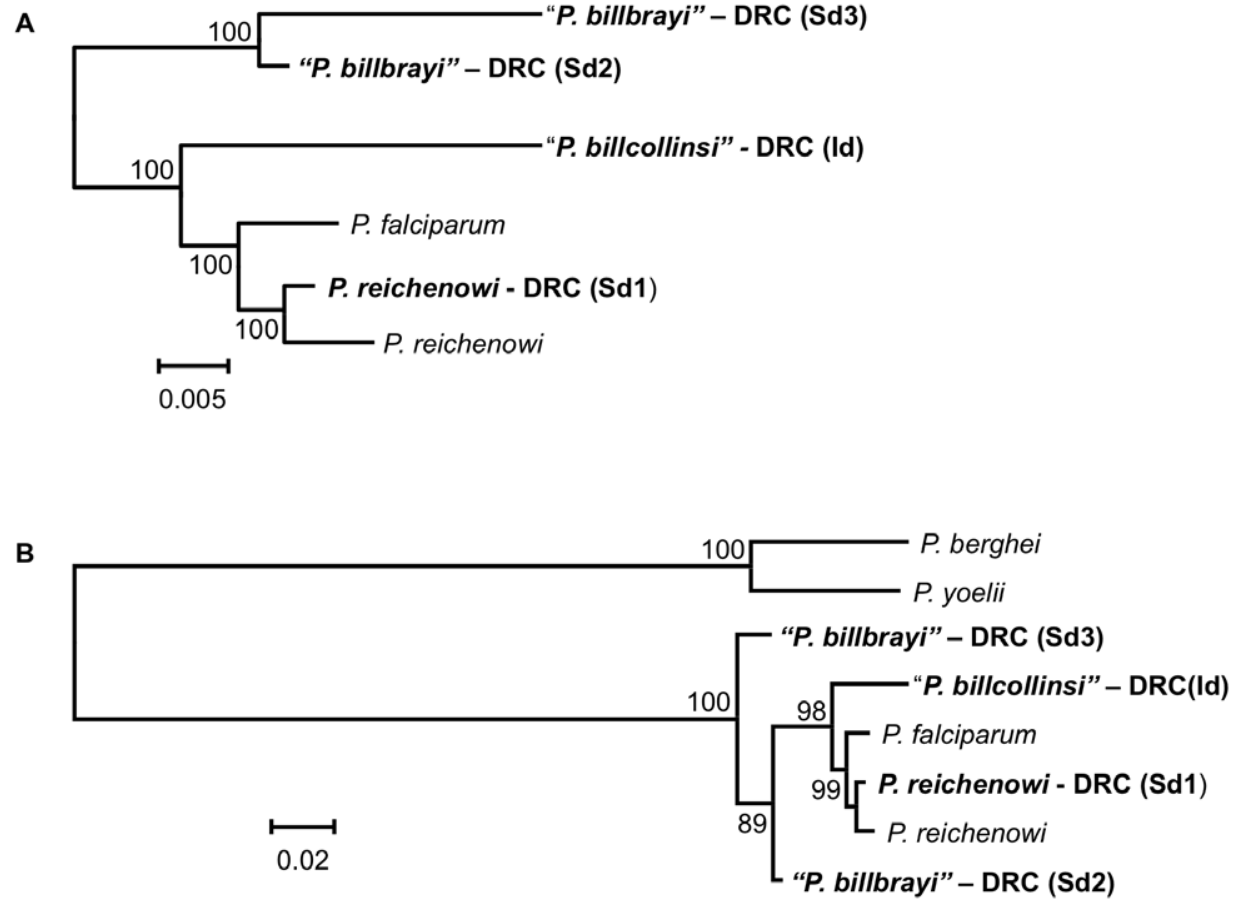

Figure 2. Phylogenetic analyses of the Laverania group based on the dhfr-ts. We report four dhfr-ts alleles, DRC (Sd1), DRC (Sd2) and DRC (Sd3) derived from the sample collected from one Ape (Shegue), and DRC (Id) derived from a sample from another Ape (Itaito). The DRC (Sd1) allele corresponds to the $P$. reichenowi sequence. In view of the similarity with the mitochondrial genome tree topology and the apparent lack of mixed species infection in the two animals from which sequences were obtained, we tentatively considered that DRC (Sd2) and DRC (Sd3) originate from $P$. billbrayi parasites, and DRC (Id) from P. billcollinsi (hence the quotation marks). Bayesian support for the nodes was inferred through a Monte Carlo Markov chain model as implemented in Mr. Bayes, with 10,000,000 generations after a "burn-in" of 3,000,000 generations. Sampling was performed every 100 generations. Mixing of the chains was properly checked after runs. Two phylogenies are presented for the gene encoding dhfr-ts. A. Phylogeny A (1789 bp), which included the P. falciparum (XM_001351443) and P. reichenowi (GQ369533, this study) dhfr-ts sequences and the four from parasites of Apes, reproduces the topology obtained from the mitochondrial genome. B. Phylogeny B (1690 bp aligned) uses rodent malarial parasites $P$. berghei and $P$. yoelii as outgroups, differs from the mitochondrial phylogeny by placing the root of the Laverania group within $P$. billbrayi alleles that are no longer monophyletic. We favour the phylogenetic hypothesis A over B since the latter is based on fewer base pairs and excludes an area with phylogenetic information among the Laverania species; such an area is not found in rodent or any other Plasmodium species so it is excluded from the phylogenetic analyses. Indeed, P. reichenowi (NC_002235 and DRC (Sd1)) cannot be clearly separated from P. falciparum indicating that rodent malarias may be too distant to serve as a reliable out-group for $d h f r$-ts. doi:10.1371/journal.ppat.1000765.g002

The parasites related to $P$. vivax-like found in chimpanzees from the DRC and Uganda might correspond to the chimpanzee parasite $P$. schwetzi. Preliminary evidence from partial $d h f r$ sequences obtained for the chimpanzees we sampled in Uganda suggests that these parasites could be related to $P$. vivax (data not shown). Unfortunately, at present a $P$. schwetz $i$ isolate is not available for comparative molecular analysis. Whether this species in Pan results from a past host switch from humans into chimpanzee, or whether it corresponds to $P$. vivax parasites recently reported in Equatorial Africa [20,21], remains a matter of speculation. It might be that the dynamics of $P$. vivax and related species in African hominids, including humans, are more complex than previously thought.

The quartan malaria parasites, $P$. brasilianum in South American primates and $P$. rodhaini in the chimpanzee, have long been considered to be strains of $P$. malariae $[13,14]$. Thus, it was interesting that the mitochondrial genomes of the parasites related to $P$. malariae found in two bonobos conform a sister clade and carry a six nucleotide insert that has not been observed for $P$. malariae or the South American parasite P. brasilianum. This could indicate that the parasites in bonobos might correspond to $P$. rodhaini, a species that would then be distinct rather than synonymous with $P$. malariae. Confirmation that this might indeed be the case awaits further molecular data from a larger set of $P$. malariae lineages from humans and Apes.
Three parasite lineages related to $P$. falciparum were found in both the chimpanzees collected from DRC and those collected from Uganda. One of these lineages clearly corresponds to $P$. reichenowi. We propose that the other lineages may represent two distinct Plasmodium species. Given the data from the near-complete mitochondrial genome sequences, and the support from $d h f r$-ts and msp2 sequences, we consider it reasonable to ascribe specific status to the parasites in the two novel lineages observed in chimpanzees. We propose to name the parasites of one of the novel lineages Plasmodium billcollinsi Krief et al. n. sp., and those of the other Plasmodium billbrayi Krief et al. n. sp., in honour of the distinguished malariologists William E. Collins and "Bill" Robert Stow Bray (1923-2008), respectively. The type material would be the mitochondrial genome sequences (holotype and paratype), with a distribution in Uganda and the DRG in Pan t. troglodytes and P. $t$. schweinfurthii as hosts.

While we were finalizing this manuscript for submission, a publication describing a novel lineage related to $P$. falciparum was reported from two Pan troglodytes sampled in Gabon [17]. Based on mitochondrial DNA sequences, the authors have also proposed that this lineage be considered a new species, $P$. gaboni [17]. When the mitochondrial sequence submitted for $P$. gaboni was compared with the mitochondrial sequence presented here, it could be concluded that $P$. gaboni and $P$. billbrayi shared a recent common 


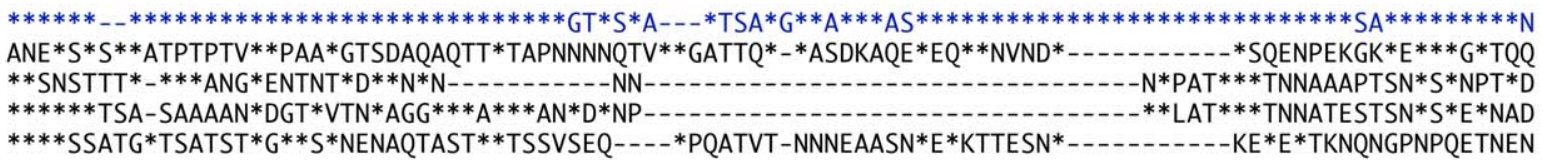

Figure 3. Alignment of the $m s p 2$ block 3 sequences obtained from Pan troglodytes sp. The predicted amino acid sequence of one member from each of the five $m s p 2$ block 3 allelic families uncovered from the Plasmodium parasites present in the chimpanzee samples. The alignment (Clusal V, DNASTAR Lasergene MegAlign version 7.2.1) comparisons were made against the only known $P$. reichenowi msp2 block 3 sequence [15], denoted "Pr" (Y14731). The msp2 block sequences obtained during our analysis were named according to the geographic origin of the samples "KNP" (Kibale National Park), followed by the sequence family (Pr for the P. reichenowi type in blue, and A to D for the others in black). Each distinct sequence found within each family was assigned a sequential number. The origins, names and accession numbers of all the msp2 block 3 sequences obtained in this study are provided in Table S1. In the alignment presented the representative sequences from the five allelic families that were included are: KNP-Pr (Prmsp2-A1, GU075719), KNP-A (msp2-KNP-A1, GU075722), KNP-B (msp2-KNP-B, GU075724), KNP-C (msp2-KNP-C1, GU075725) and KNP-D (msp2-KNP-D, GU075726). Stars $\left(^{*}\right)$ represent residue similarity and dashes $(-)$ represent gaps. doi:10.1371/journal.ppat.1000765.g003

ancestor (Fig. S1). However, the differences were of sufficient importance (e.g. P. gaboni has a unique insert) to lead us to consider $P$. gaboni as a possible other additional member of the Laverania clade. Nonetheless, this assessment is at present mitigated by the fact that the contiguous mitochondrial sequence provided for the $\mathrm{K}$ isolate of $P$. gaboni (GenBank Accession No FJ895307) was assembled from discontinuous fragments that were amplified separately, hence the unavoidable gaps. Furthermore, if the animal from which the sample was obtained harboured a mixed infection, as did many of the chimpanzees that we sampled, the different fragments used for assembly might have originated from different species or lineages. Consequently, we opted not to consider the P. gaboni mitochondrial sequence in our phylogenetic analyses until such a time that the mitochondrial sequence from this lineage is confirmed, a view also adopted by Rich et al. [12].

We are aware that the validity of a species described only by sequences of one or more genes is open to debate, as this does not conform to current acceptable criteria. It would have been desirable to obtain some morphological data to provide a classical description of a novel species. The description of a new Plasmodium species is classically made after microscopic examination of Giemsa-stained infected erythrocytes, most often showing all asexual and sexual developmental stages. In some cases, it is necessary to examine the form of the parasite in the insect vector and/or during the hepatic stages, while for others differentiation from known species requires establishing one or more biological characteristics such as host specificity, the course of infection, or the ability to breed true. In the case of Plasmodium parasites that infect highly protected hosts (such as chimpanzees, gorillas and orang-utans) invasive sampling is highly restricted. On rare occasions it is possible to obtain a blood sample, but experimental infections of such animals are now nearly universally legally proscribed. Thus, the likelihood to obtain the morphological and biological data required to define and name a novel Plasmodium species for such hosts is highly remote. Furthermore, the presence in a single sample of multiple species would make it difficult to derive reliable conclusions from observations of a few blood smears. This is further exacerbated when parasite levels are low because this restricts microscopic examination to a few forms in thick smears where parasite morphology is poorly preserved. In our case, the six chimpanzees we sampled had low parasite loads, and four of them had mixed species infections. Had we had the opportunity to examine blood smears, a crescent-shaped gametocyte distinctive of $P$. falciparum and $P$. reichenowi might have been observed, but it would not have been possible to ascribe it with any degree of confidence to any one of the lineages detected by PCR amplification. Therefore, in the case of blood dwelling protozoan parasites of African Apes or other protected species, molecular data become the only accessible and reliable taxonomic features. In our study, we have considered that the phylogenetic analysis and genetic diversity comparisons based on the near-complete mitochondrial genomes, combined and supported with similar data from two nuclear genes, provided sufficient grounds to propose the description of two new species. The fact that similar sequence analyses correctly predict the specific status of wellestablished Plasmodium species (Fig. 1 and Table 1), adds to our confidence in the validity of $P$. billbrayi and $P$. billcollinsi as bona fide species. We nonetheless consider that it would be worthwhile for the community to agree on standardized parameters derived from defined molecular data that could serve to describe Plasmodium species for which no morphological or biological data are likely to become available.

The findings we present in this manuscript advocate a reappraisal of current views on the evolution and origin of $P$. falciparum. When it was thought that $P$. reichenowi and $P$. falciparum were unique among all primate malaria parasites, two hypotheses for the origin of $P$. falciparum as a parasite of humans were considered: co-speciation in their respective hosts, or a host switch followed by independent evolution. Grounds for favouring one hypothesis over the other shifted with time, as the weight of evidence that could support one hypothesis over the other was limited, principally by the availability of only a single $P$. reichenow $i$ isolate. Recent analyses of data from parasites sampled from eight chimpanzees provided clear support for the host-switch scenario [12]. The data we present further support this finding and provide a more detailed account of the events leading to the origin of $P$. 


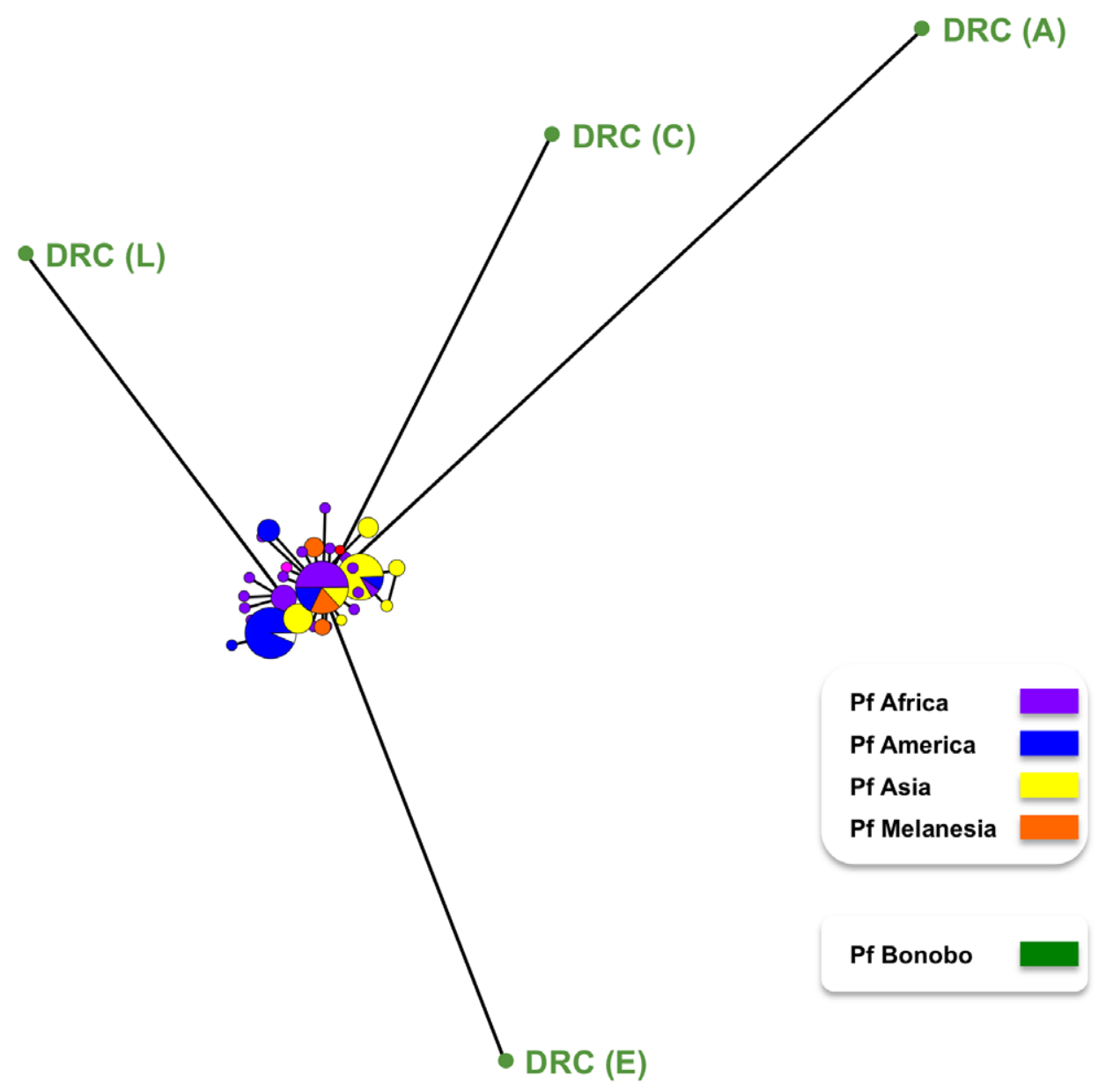

Figure 4. Mitochondrial haplotype map for $P$. falciparum populations found in humans and in bonobos. The mitochondrial genomes from parasite lines collected from humans [9] and of the four obtained from parasites in bonobos, DRC (A), DRC (C), DRC (E) and DRC (L), were used to obtain the haplotype network presented. It was inferred under a median joining algorithm with posterior pruning using maximum parsimony criteria as implemented in Network 4.1.1.2 [42]. The size of the circles is proportional to the haplotype frequency with each colour indicating which were derived from $P$. falciparum collected from bonobos, and the geographical origin of the sequences from $P$. falciparum collected from humans. doi:10.1371/journal.ppat.1000765.g004

falciparum as a parasite of humans. When the tree topologies derived from the $d h f r$-ts and mitochondrial sequences (Fig. 1 \& Fig. 2) are considered, the most parsimonious interpretation is that $P$. falciparum belongs to a monophyletic group of malarial parasites that have evolved in African Apes. We proceeded to estimate the divergence time of the most recent common ancestor for the Laverania clade. We agree that the use of molecular clocks is not without pitfalls, even when good time points can be used for calibration $[22,23]$. In the particular case of parasitic organisms, an assumption of some level of host specificity (though not necessarily co-speciation) is needed in order to use host evolution for estimating the parasite mutation rates. Therefore, we estimated times of divergence of the mitochondrial sequences using models that allow the use of relaxed molecular clocks [24]. Although the Homo/Pan divergence time has been commonly used as a point of calibration for the falciparum-reichenowi divergence (e.g. $[9,17])$, we excluded it in order to avoid circularity in the analyses. Thus, we estimated the mutation rates under two previously used scenarios: a), the Plasmodium spp. currently found in macaques radiated with their primary hosts, the genus Macaca [5], and b) P. gonderi, a parasite from African monkeys, and macaque parasites codiverged when Macaca branched from other Papionina [25]. It is worth noting that neither of these two time points requires co- speciation (i.e. where specific malarial parasites co-speciate with specific non-human primate lineages generating phylogenies with identical topologies), but simply that several malarial parasites started their radiation with a major groups of non-human primates allowing for extensive host-switches. Such timeframes can be estimated even in the absence of good phylogenetic trees [26].

It is interesting that our time estimates (Table 2) that did not use the Homo-Pan divergence as a calibration point, were not substantially different from those estimated by others [9,17] who used the $P$. falciparum - $P$. reichenowi divergence assuming cospeciation with Homo-Pan. The estimates of the divergence times for the Laverania clade members (Table 2) indicated that all the four lineages might have originated between 6.0 and 19 million years ago (Mya). Regardless of the wide confidence interval, this time frame is consistent with the origin of the genus Pan, but it clearly indicated that the Laverania lineages may have started to diverge long before the divergence Pan-Homo [27]. In addition, the phylogeny clearly indicates that the human parasite, $P$. falciparum, is the only Homo parasite among several Pan species in the Laverania clade. Given the phylogeny, a Pan host appears as an ancestral characteristic of the lineage. Therefore, when both phylogenies and estimated times of divergence are considered, a co-evolutionary origin of $P$. falciparum as a parasite of humans can 
Table 2. Estimated TMRCA for different parasite groups.

\begin{tabular}{ll}
\hline Clade & \\
\hline P. falciparum in Pan paniscus & $\begin{array}{l}\text { TMRCA mean Mya } \\
\text { (L 95\% Cl-H 95\% CI) }\end{array}$ \\
\hline P. falciparum in H. sapiens & $0.77(0.43-1.6)$ \\
P. falciparum in Pan paniscus and H. sapiens & $0.20(0.078-0.33)$ \\
P. reichenowi & $0.85(0.46-1.3)$ \\
P. reichenowi - P. falciparum in Pan paniscus & $1.8(0.60-3.2)$ \\
P. billbray n. sp. & $2.2(1.0-3.1)$ \\
P. billcollinsi $\mathbf{n}$. sp. & $1.1(0.52-1.7)$ \\
Laverania radiation & $0.97(0.38-1.7)$ \\
\hline
\end{tabular}

doi:10.1371/journal.ppat.1000765.t002

be confidently excluded. Consequently the hypothesis that $P$. falciparum originated as a result of a host-switch between humans and Apes becomes favoured. However, our data indicate more complex scenarios that can only be addressed when data from multiple isolates of the parasite lineages currently present in both the hosts involved are included in the analyses.

The mitochondrial haplotype map (Fig. 4) provides evidence that the sub-population of four $P$. falciparum parasites in bonobos were genetically more diverse that of the extensive $P$. falciparum population in humans available to date. The most parsimonious interpretation of this line of evidence is that P. falciparum originated as a human parasite via a host-switch from Pan paniscus. When the human P. falciparum mitochondrial sequences alone are considered, our estimate of the time to the most recent common ancestor (TMRCA) was 78,000-330,000 years ago. While we cannot rule out that the available sample of $P$. falciparum mitochondrial genomes properly represent the genetic diversity of the species, this time frame is consistent with one expected for a parasite expanding early in human history. However, when considered together, the two distinct $P$. falciparum populations of humans and bonobos are estimated to have diverged from other members of the Laverania clade between 1.0 and 3.1 Mya. This timeframe coincides with the divergence of bonobo from the common chimpanzee $[28,29]$. The estimated TMRCA of 0.4 to $1.6 \mathrm{Mya}$ for the P. falciparum found in bonobos coincides with the origin of bonobos [28]. Taken together, our analyses indicate that $P$. falciparum, as a species, has long been associated with Pan paniscus and only subsequently switched into humans. The topology of the mitochondrial haplotype network (Fig. 4) is consistent with this interpretation and suggests that few lineages expanded in the human population after this event. The parasites we obtained over a short period from a single bonobo community probably constitute a biased sample set. A reliable estimate of the timing for the host-switch and the number of times this event might have taken place would require the inclusion of sequences from a larger set of $P$. falciparum parasites from bonobos from diverse locations. Assuming that there was no sampling bias with respect to the $P$. falciparum populations collected by others from humans, the limited data from bonobo parasites we present here can be most conservatively interpreted to support a single switching event, though it does not allow excluding multiple events. It is also possible that host switching still occurs today in areas where humans and bonobos are in close epidemiological contact. The presence of double or triple mutations associated with resistance to pyrimethamine in the four $d h f r$ sequences obtained for the $P$. falciparum of bonobos is consistent with this, because these mutations are common in P. falciparum collected in 2008 from residents around Kinshasa [30]. At present, we cannot rule out the possibility that these $d h f r$ mutations might have been selected independently in bonobos during the three months treatments with Bactrim $^{\text {TM }}$ (trimethoprim + sulfamethoxazole, two drugs that target the same enzymes of the folate pathway as the antimalarial combination of pyrimethamine and sulfadoxine) to which apes in the sanctuary were occasionally subjected. Finally, it could be speculated that the parasites in bonobos and in humans have recombined sexually.

The scenario we propose for the origin of $P$. falciparum in humans differs in several respects from a very recently formulated hypothesis that proposed that this species originated from a single transfer of $P$. reichenowi from chimpanzees to humans [12]. These conclusions were based on the analysis of the genetic diversity and tree topologies derived from fragments of the mitochondrial cytochrome b gene (528 bp), the apicoplast caseinolytic protease (316 bp), and the nuclear small subunit ribosomal RNA gene (371 bp), obtained from eight Plasmodium-infected chimpanzees (three from Pan $t$. verus, and five from Pan $t$. troglodytes). One assumption was that these sequences were derived from a single parasite specie, $P$. reichenowi, found in Pan troglodytes sp. This was a fair supposition to make since these short sequences did not provide sufficient resolution to distinguish their lineages from that of the only known P. reichenowi isolate. However, when these partial cytochrome $b$ sequences are compared to the homologous region in the mitochondrial genomes that we obtained, there are clear indications that some might correspond to $P$. reichenowi, but also that most cluster either with the $P$. billbrayi or the $P$. billcollinsi lineages reported here (Fig. S1), which differ to such an extent from $P$. reichenowi that they could be considered as distinct species. Indeed this is evident on examination of the topology and branch lengths in the phylogenetic tree presented for the cytochrome $b$ fragment (see Fig. 4 of [12]), where the eight isolates cluster into three groups removed from $P$. reichenowi. Our data provides evidence of a contrasting and more complex evolutionary scenario where P. falciparum evolved as a species in bonobos (Pan paniscus) where it was one of at least four parasite species that radiated in the genus Pan before it switched into humans.

The infections of bonobos by $P$. falciparum were not associated with any overt clinical signs, nor would the levels of parasitaemia have allowed detection by microscopy, suggesting a state of chronic malaria typical of infections in natural hosts. This is consistent with previous observations, including some made on splenectomised chimpanzees with high parasite levels [13,14], in which chimpanzees experimentally infected with various parasite species including $P$. falciparum showed few clinical signs whether at peak parasitaemias or during the subsequent lengthy chronic infections $[13,14]$. This minor impact on the health of chimpanzees was recently supported by the failure to detect a signature of positive selection in their G6PD genes, despite a long association with Plasmodium parasites [31]. The contrasting parasitological and clinical evolutions of $P$. falciparum in its two hosts, humans and bonobos, which have highly similar genomes, provides an excellent opportunity for comparative genomic studies to uncover the genetic or molecular basis for its higher virulence in humans. Such knowledge could be exploited to devise novel approaches to reduce the substantial global morbidity and mortality burdens.

It is likely that bonobos, in which we have found significant numbers to be naturally infected with $P$. falciparum or P. malariae, are also susceptible to infections by $P$. ovale and $P$. vivax, as is the case for chimpanzees [13,14,19]. One can now, therefore, justifiably explore whether bonobos and chimpanzees could act as a reservoir for all Plasmodium species that afflict humans. The 
potential impact of zoonotic malaria transmission on human health has been recently exemplified by a stable focus of potentially lethal $P$. knowlesi, a benign parasite of macaques, in inhabitants of Malaysian Borneo [32,33]. Such a possibility has not been considered for sub-Saharan Africa. A zoonotic reintroduction of malaria into communities that live in hyperendemic areas is likely to be of little consequence. However, this would hinder efforts to eradicate malaria and might possibly lead to epidemic foci in formerly malarious regions whose inhabitants have lost immunity acquired against malaria. Furthermore, humans have been shown to be susceptible to infection by two of the parasite species of African Apes ( $P$. rodhaini and $P$. schwetzi) $[13,14]$, and the meagre data available does not exclude the possibility that humans can be infected by $P$. reichenowi or the two new species we describe here. Using the sequence data we obtained from chimpanzee parasites, it will now be possible to seek these parasites in groups of humans that are in contact with African Apes.

In conclusion, the data gathered from a limited molecular analysis of a modest number of chimpanzee blood samples have not only significantly added to our knowledge of Plasmodium in our closest relatives, bonobos and chimpanzees, but also provided tantalizing insights into the evolutionary history of the malaria parasites of humans. We urge the scientific and the wildlife conservation communities to devote some resources to archive the parasites of Great Apes, which are at present likely to remain only amenable to molecular investigations, and to develop in vitro and/ or ex-vivo methods to preserve and maintain them. These studies might provide novel approaches that could help control and eventually eradicate pathogens that have long exacted devastating global health, economic and social burdens.

\section{Methods}

\section{Samples}

Ethics statement. Collections of blood samples from animals in the DRC were made during routine annual medical check-ups. Authorization for the samples collected in the DRC was obtained from the Direction de la Conservation de la Nature et Organe de gestion de la CITES at the Ministère de l'Environnement, Conservation de la nature et Tourisme (DR), and the use of samples for scientific investigations was approved (CITES E0909/ 07). Specific authorization was also granted to "Les Amis des Bonobos du Congo" by the Ministère de la Recherche Scientifique (DRC). The few drops of blood from the chimpanzees at the Kibale National Park (Uganda) were obtained non-invasively: one in the course of post-mortem examination, the others from blood that dripped from wounds; collection of blood samples from the chimpanzees on Ngamba Island Chimpanzee Sanctuary (Uganda) were also conducted during routine annual medical check-ups; DNA extraction and preliminary PCR analysis were performed in Uganda. Authorization to use the DNA extracted from the samples for the purposes of genetic analyses of Plasmodium parasites that might be present was granted by the Uganda Wildlife Authority and the Uganda National Council for Science and Technology. The animal work was conducted according to relevant national and international guidelines. In all cases, the animals were not subjected to any experimental procedures, and the blood samples were obtained from aliquots collected independently by veterinarians carrying out routine medical examination. After consideration of the protocols of the study, the Arizona State University Institutional Review Board considered that the proposed molecular analyses of parasite DNA did not require formal approval. The Institutional Review
Board of the Muséum National d'Histoire Naturelle also considered it unwarranted to seek formal approval for the genetic analysis of parasites present in material collected noninvasively and/or in an aliquot of samples collected during routine medical care of animals.

Chimpanzees, Uganda. Blood samples were collected on EDTA from three, wild, eastern chimpanzees (Pan troglodytes schweinfurthii), members of the Kanyawara community in Kibale National Park in western Uganda. A team led by S. Krief closely monitors the behaviour and health status of the Kanyawara chimpanzees. The blood samples have been opportunistically collected from one adult female (named NL) found dead on 20 Jan 2007 , and from blood that dripped from wounds of an adolescent female (named JK) found caught in a snare on the 24 Oct 2006, and from those of another adolescent female (named OK) found injured on 30 Sep 2006. The samples were kept at $-80^{\circ} \mathrm{C}$ until DNA extraction.

Blood samples were collected on EDTA in 2005 from thirtyeight semi-captive chimpanzees (Pan troglodytes schweinfurthii) at the Ngamba Island Chimpanzee Sanctuary situated on Lake Victoria close to Kampala in Uganda. The blood was collected under general anaesthesia during the routine annual health check monitoring. The samples were kept at $-80^{\circ} \mathrm{C}$ at the Uganda Virus Research Institute (Entebbe, Uganda) until DNA extraction.

Chimpanzees, DRG. Eight orphan Pan troglodytes troglodytes from the DRC were sampled immediately after rescue between 2003 and 2006.

Bonobos, DRG. Blood samples were collected from 42 bonobos (Pan paniscus) that were kept at the Lola ya Bonobo Sanctuary, on the outskirts of Kinshasa in the Democratic Republic of Congo. The samples were obtained in 2007 as part of the routine annual health monitoring of 20 females and 22 males (age from 2 to 22 years old). The health status of each animal was scored on a scale of 1 to $3(1=\operatorname{good} ; 2=$ medium; $3=$ bad). 21 animals were scored 1, 18 were scored 2 and three were scored 3. Cough symptoms were noted in 19 individuals. Body temperatures ranged from $35.4^{\circ} \mathrm{C}$ to $37.7^{\circ} \mathrm{C}$, but neither of these two parameters were correlated with the health score, nor with the presence of Plasmodium as detected by PCR. None of the animals suffered from diarrhoea, nor was blood found in the urine samples collected.

\section{DNA extraction, amplification protocols and sequencing strategies}

For all samples, genomic DNA was extracted from aliquots of $200 \mu \mathrm{l}$ of whole blood using the Qiagen DNeasy Blood and Tissue Kit (Qiagen, Germany), and the DNA obtained resuspended in $200 \mu \mathrm{l}$ of buffer. Blood smears were not available for microscopic examination, thus parasite levels were estimated using PCR analysis of a 10-fold serial dilution series of the DNA purified from the positive samples. The nested PCR detection assay used was based on the small subunit ribosomal RNA gene (ssrRNA), using oligonucleotide primers that were specific to, and conserved in, all known Plasmodium species [18]. This established that the parasite burdens in these animals were very low $(<10-100$ parasite per $\mu \mathrm{l}$ of blood, and in one case $<1000$ parasites per $\mu \mathrm{l}$ ).

Approximately $5,800 \mathrm{bp}$ (out of 6,000) of the parasites' mitochondrial genome were amplified using the oligos Forward 5'-GAGGATTCTCTCGACACTTCAATTCGTACTTC and Reverse 5'-CAGGAAAATWATAGACGGAACCTTGGACTC with Takara LA Taq ${ }^{\mathrm{TM}}$ Polymerase (TaKaRa Takara Mirus Bio), (1 cycle $94^{\circ} \mathrm{C}$ for $1 \mathrm{~min}$, then 30 cycles of $94^{\circ} \mathrm{C}$ for $30 \mathrm{sec}$ and $68^{\circ} \mathrm{C}$ for $7 \mathrm{~min}-1$ cycle $72^{\circ} \mathrm{C}$ for $\left.10 \mathrm{~min}\right)$. PCR products were cloned in the PGem ${ }^{\circledR}-\mathrm{T}$ vector (Promega). In the case of the 
mitochondrial genome, we report sequences deposited in GenBank (Accession numbers are in parentheses following species name) for the Asian macaque parasites $P$. inui strain Taiwan II (GQ355483), P. inui strain Leaf Monkey II (GQ355482), and for $P$. brasilianum (GQ355484) from South American primates. Other sequences were reported in other studies: $P$. inui Mulligan (AB354572), $P$. fieldi (AB354574), P. simiovale (AB434920, AY800109), P. knowlesi (NC_007232), P. cynomolgi (AY800108), P. fragile (AY722799) and $P$. coatneyi (AB354575); P. hylobati (AB354573) from gibbons, P. simium (AY800110), P. gonderi from African monkeys (AY800111), and the parasites of humans $P$. ovale (AB354571) and P. malariae (AB354570). Additional information about these species, including their description, basic biology, geographic distribution and host-range can be found elsewhere [13]. Additional sequences of Plasmodium mitochondrial genomes were obtained from the GenBank (Accession numbers are in parentheses following species name): the avian malarial parasites $P$. gallinaceum (NG_008288), P. juxtanucleare (NG_008279), and $P$. relictum (AY733088-AY733090); the rodent malarial parasites $P$. yoelii (M29000), P. berghei (AF014115), P. chabaudi (AF014116); the non-human primate malarial parasite $P$. reichenowi (NC_002235); the human malarial parasite $P$. falciparum (AY282930) and $P$. vivax (AY598140). The avian parasite Leucocytozoon sabrazesi (NG_009336) was used as outgroup.

The gene encoding dihydrofolate reductase-thymidylate synthase $(d h f r-t s)$ from $P$. falciparum or related species in samples collected from chimpanzees in Uganda and bonobos in the DRC was obtained as two overlapping fragments amplified by nested PCR using the following primer pair for the primary reaction: Pfdhfrts-F 5'-ATGATGGAACAAGTCTGGGACGTTTTCG and Pfdhfrts-R 5'-GGAGGGATATCGATTGAAATTTTTTCATG, $\left(2.5 \mathrm{mM} \mathrm{Mg}{ }^{2+}\right.$, annealing at $58^{\circ} \mathrm{C}$ ) The two separate secondary reactions were initiated with $1 \mu \mathrm{l}$ of the product from the primary reaction using the following primer pairs Pfdhfrts-F and Pfdhfrts-NR 5'-GGGAAATATTGACTTAAATCAAATTTC $\left(1.5 \mathrm{mM} \mathrm{Mg}^{2+}\right.$, annealing at $\left.58^{\circ} \mathrm{C}\right)$ that amplifies the fragment coding for the DHFR and linker domains, or Pfdhfrts-NF 5'CAAAGTGATGGAAGGgGaGTAGGTG and Pfdhfrts-R $\left(3.5 \mathrm{mM} \mathrm{Mg}^{2+}\right.$, annealing at $58^{\circ} \mathrm{C}$ ) that amplifies the fragment encoding the TS domain. All reactions were initiated with $1 \mu \mathrm{l}$ of template (equivalent to ca. $1 \mu \mathrm{l}$ of whole blood) in a total reaction volume of $40 \mu \mathrm{l}$ (final concentrations of $125 \mu \mathrm{M}$ dNTP, $250 \mathrm{nM}$ of each oligo, and 2 units $/ 100 \mu \mathrm{l}$ AmpliTaq polymerase), with the following cycling conditions: $95^{\circ} \mathrm{C}$ for $5 \mathrm{~min}$, then 30 cycles of 2 min annealing (see above for temperatures used for each primer set), 2 min extension at $72^{\circ} \mathrm{C}$ and $1 \mathrm{~min}$ denaturation at $94^{\circ} \mathrm{C}$, after a final annealing step followed by a 5 min extension step, the reaction temperature was brought down to $25^{\circ} \mathrm{C}$ before storage at $-20^{\circ} \mathrm{C}$.

The gene encoding the $d h f r$-ts from parasites related to $P$. falciparum in samples collected from chimpanzees in the DRC, was amplified using the primers: Forward 5'-ATGATGGAACAAGTCTGCG and Reverse 5'-TTAAGCAGCCATATCCATTG. The PCR conditions were: a partial denaturation at $94^{\circ} \mathrm{C}$ for $3 \mathrm{~min}$ and 35 cycles with $1 \mathrm{~min}$ at $94^{\circ} \mathrm{C}, 1 \mathrm{~min}$ at $53^{\circ} \mathrm{C}-$ $55^{\circ} \mathrm{C}$ and $2 \mathrm{~min}$ extension at $72^{\circ} \mathrm{C}$, a final extension of $10 \mathrm{~min}$ was added in the last cycle. Aligning dhfr-ts sequences among distantly related species of Plasmodium was difficult due to several insertions-deletions. We performed two analyses, one including only $P$. falciparum-like sequences on $1789 \mathrm{bp}$ and a second including P. gallinaceum (AY033582), P. chabaudi (M30834), and P. yoelii (XM_719562) with only 1690 bp.

The fragment encoding the block 3 polymorphic domain of merozoite surface protein 2 (msp2) from P. falciparum or related species in samples collected from chimpanzees in the Uganda and bonobos in the DRC was by nested PCR amplification using the following primer pairs: primary reaction M2-P1 5'-GAAGGTAATTAAAACATTGTC and M2-P2 5'-GAGGGATGTTGCTGCTCGACAG, and a secondary reaction were initiated with $1 \mu \mathrm{l}$ of the product from the primary reaction using M2-N1 5'CTAGAACCATGCATATGTCG and M2-N2 5'-GAGTATAAGGAGAAGTATG. All reactions were initiated with $1 \mu \mathrm{l}$ of template in a total reaction volume of $40 \mu \mathrm{l}$ (final concentrations of $1.0 \mathrm{mM} \mathrm{Mg}{ }^{2+}, 25 \mu \mathrm{M} \mathrm{dNTP}, 250 \mathrm{nM}$ of each oligo, and 2 units $/ 100 \mu \mathrm{l}$ AmpliTaq polymerase), with the following cycling conditions: $95^{\circ} \mathrm{C}$ for $5 \mathrm{~min}$, then 30 cycles of $30 \mathrm{sec}$ annealing at $50^{\circ} \mathrm{C}, 1$ min extension at $72^{\circ} \mathrm{C}$ and 30 sec denaturation at $94^{\circ} \mathrm{C}$, after a final annealing step followed by a 5 min extension step, the reaction temperature was brought down to $25^{\circ} \mathrm{C}$ before storage at $-20^{\circ} \mathrm{C}$.

In the majority of cases these sequences were derived from two or more independent amplifications. All the sequences obtained and reported here were submitted to GenBank (Accession numbers and the corresponding gene fragments are presented in the Table S1).

\section{Phylogenetic analyses}

Initial Neighbor Joining (NJ) trees were inferred under Tamura3P model of nucleotide substitution [34] in Mega4 [35]. Maximum likelihood (ML) search of a tree topology was implemented in PAML4 [36] under a General Time Reversible $($ GTR $)+\mathrm{I}+\Gamma_{4}$ substitution model, chosen based on likelihood ratio tests [37], and employing the NJ method to generate an initial tree. Bayesian support for the nodes was inferred in MRBAYES [38], under a General Time Reversible (GTR) + I + $\Gamma_{4}$ substitution model, using 4 Markov chains and 10,000,000 Markov Chain Monte Carlo (MCMC) steps, discarding the first $3,000,000$ steps $(30 \%)$ as a burn-in. Sampling was performed every 500 generations. Mixing of the chains and convergence was properly checked after runs. The recovered ML and Bayesian trees were identical.

Although a total of eight distinct near-complete mitochondrial genomes were obtained from the parasites found in the bonobos, we stringently excluded any where the accuracy of the sequence obtained was not optimal, thus only 4 sequences were included in the phylogenetic and other analyses.

\section{Estimation of divergence times}

The mutation rates that have been widely used in Plasmodium evolutionary genetic studies have used the Homo/Pan divergence time as a point of calibration for the falciparum-reichenowi divergence (for e.g. [9,39]). However, using such rates will make whatever argument we put forward about the origin of $P$. falciparum and $P$. reichenowi circular. Thus, in order to avoid tautological arguments, we estimated mutation rates by considering time of divergence under two scenarios: i) the Plasmodium currently found in macaques radiated with the genus Macaca [5], which allows the estimation of a substitution rate of 2.83E-09 subs/site/year; ii) assuming that $P$. gonderi and macaque parasites co-diverged when Macaca branched from other Papionina [25], which allows the estimation of a mutation rate of 5.07E-09. It is worth noting that these mutation rates were not particularly off other estimates obtained for Plasmodium mitochondrial genomes (for e.g. [9]) indicating that, at least as first approximations, these scenarios are reasonable.

We employed a Bayesian approach with a relaxed clock [40] as implemented in BEAST [24]. The estimations of times of divergence for the clades of interest were performed by running 4 independent runs of 10,000,000 Markov Chain Monte Carlo 
(MCMC) steps after discarding the first 30\% of the steps as burnin, and sampling being performed every 1,000 steps. Previous runs showed that this burn-in was sufficient for the chains to reach stationary distribution. For the relaxed version of the clock we assumed a lognormal distributed clock for the mutation rate, with an average mutation rate according to each scenario mentioned in the previous paragraph, under a Yule prior for the simulation of the lineages during tree reconstruction. Results of the runs were analyzed with Tracer v1.4 [41] and estimates of average divergence times and confidence intervals were recovered. We checked the adequate mixing of the MCMC chains for each run in and the effective sample size of the estimates, making sure that all of them were above 100 . The runs were combined in Tracer to generate the final estimates of time of divergence and their $95 \%$ confidence intervals.

\section{GenBank Accession numbers submitted with this manuscript}

The following sequences were submitted to the GenBank: Nearcomplete Plasmodium mitochondrial genomes from parasites of chimpanzees, bonobos and other primate hosts GQ355468GQ355486; msp2 block 3 from parasites collected from Pan $t$. schweinfurthii (Uganda) GU075719-GU75726, and from parasites collected from Pan t. troglodytes (DRG) GU131994-GU131995; $d h f r$-ts sequences from parasites collected from Pan $t$. troglodytes (DRG) GQ369532-GQ369536; P. falciparum msp2 block 3 sequences from bonobo samples GU075709-GU075718; $P$. falciparum dhfr-ts partial sequences from bonobo samples GQ859592-GQ859595).

\section{Supporting Information}

Figure S1 Phylogenetic tree of Plasmodium based on a cytochrome $b$ fragment. NJ tree on $520 \mathrm{bp}$ of cytochrome $b$ using Tamura 3 parameter model, 1000 bootstrap pseudo-replications.

\section{References}

1. Escalante AA, Ayala FJ (1995) Evolutionary origin of Plasmodium and other Apicomplexa based on rRNA genes. Proc Natl Acad Sci USA 92: 5793-5797.

2. Qari SH, Shi Y-P, Pieniazek NJ, Collins WE, Lal AA (1996) Phylogenetic relationship among the malaria parasites based on small subunit rRNA gene sequences: monophyletic nature of the human malaria parasite, Plasmodium falciparum. Mol Phylogenet Evol 6: 157-165.

3. Escalante AA, Barrio E, Ayala FJ (1995) Evolutionary origin of human and primate malarias: evidence from the circumsporozoite protein gene. Mol Biol Evol 12: 616-626.

4. Escalante AA, Freeland DE, Collins WE, Lal AA (1998) The evolution of primate malaria parasites based on the gene encoding cytochrome $b$ from the linear mitochondrial genome. Proc Natl Acad Sci USA 95: 8124-8129.

5. Escalante AA, Cornejo OE, Freeland DE, Poe AC, Durrego E, et al. (2005) A monkey's tale: the origin of Plasmodium vivax as a human malaria parasite. Proc Natl Acad Sci USA 102: 1980-1985.

6. Perkins SL, Schall JJ (2002) A molecular phylogeny of malarial parasites recovered from cytochrome b gene sequences. J Parasitol 88: 972-978.

7. Snow RW, Guerra CA, Noor AM, Hla Yin Mint, Hay SI (2005) The global distribution of clinical episodes of Plasmodium falciparum malaria. Nature 434: 214-217.

8. Conway DJ, Fanello C, Lloyd JM, Al-Joubori BMA-S, Baloch AH, et al. (2000) Origin of Plasmodium falciparum malaria is traced by mitochondrial DNA. Mol Biochem Parasitol 111: 163-171.

9. Joy DA, Feng X, Mu J, Furuya T, Chotivanich KT, et al. (2003) Early origin and recent expansion of Plasmodium falciparum. Science 300: 318-321.

10. Rich SM, Licht MC, Hudson RR, Ayala FJ (1998) Malaria's Eve: evidence of a recent population bottleneck throughout the world populations of Plasmodium falciparum. Proc Natl Acad Sci USA 95: 4425-4430.

11. Volkman SK, Barry AE, Lyons EJ, Nielsen KM, Thomas SM, et al. (2001) Recent origin of Plasmodium falciparum from a single progenitor. Science 293: 482-484.

12. Rich SM, Leendertz FH, Xu G, Lebreton M, Djoko CF, et al. (2009) The origin of malignant malaria. Proc Natl Acad Sci USA.

13. Coatney GR, Collins WE, Warren M, Contacos PG (1971) The primate malarias. Washington DC: U.S. Government Printing Office. pp 1-366.
Haplotypes represented in bold are as follows: Plasmodium species that infect humans (black), the haplotypes we present in the manuscript (blue), the haplotype proposed as P. gaboni by Ollomo et al. [17] (purple), and the haplotypes presented as P. reichenowi by Rich et al. 2009 [12] (red). The species we propose, P. billbrayi and $P$. billcollinsi, are clearly set apart despite the relatively poor resolution inherent to using a short DNA sequence. For the "reichenowi-" haplotypes (red), "reichenowi-Rafikil" and "reichenowi-Rafiki2" might belong to $P$. reichenowi, but three of the others cluster closely with $P$. billcollinsi and another three cluster more loosely with the $P$. billbrayi/P. gaboni group.

Found at: doi:10.1371/journal.ppat.1000765.s001 (0.35 MB PDF)

Table S1 Origin of the blood samples that yielded Plasmodium sequences (name in bold and GenBank Accession number in parentheses).

Found at: doi:10.1371/journal.ppat.1000765.s002 (0.09 MB PDF)

\section{Acknowledgments}

We are very grateful to the Uganda Wildlife Authority and the Uganda National Council for Science and Technology for granting us permission to conduct this research. We also extend our gratitude to Makerere University Biological Field Station for logistic support they provided. We are thankful to the Field Assistants, Japan Musinguzi and Ronald Musinguzi for their help during the fieldwork. LR and GS are currently part of an official collaboration between SIgN/A*STAR and INSERM (Laboratoire International Associé, INSERM)

\section{Author Contributions}

Conceived and designed the experiments: SK AAE MAP MC OEC ACG TFM LR GS. Performed the experiments: SK AAE MAP LM OEC JMC CL FL ACG GS. Analyzed the data: SK AAE MAP CA MH AF JMK JMK MC OEC JMC CL ACG TFM LR GS. Contributed reagents/ materials/analysis tools: SK AAE MAP LM CA MH AF JMK JMK MC OEC JMC CL FL ACG TFM LR GS. Wrote the paper: SK AAE MAP OEC GS.

14. Garnham PCC (1966) Malaria parasites and other haemosporidia. Oxford: Blackwell Scientific Publications. pp 1-1114.

15. Dubbeld MA, Kocken CHM, Thomas AW (1998) Merozoite surface protein 2 of Plasmodium reichenowi is a unique mosaic of Plasmodium falciparum allelic forms and species-specific elements. Mol Biochem Parasitol 92: 187-192.

16. Tanabe K, Sakihama N, Hattori T, Ranford-Cartwright LC, Goldman IF, et al. (2004) Genetic distance in housekeeping genes between Plasmodium falciparum and Plasmodium reichenowi and within P. falciparum. J Mol Evol 59: 687-694.

17. Ollomo B, Durand P, Prugnolle F, Douzery E, Arnathau C, et al. (2009) A new malaria agent in African hominids. PLoS Pathog 5: e1000446. doi:10.1371/ journal.ppat. 1000446.

18. Snounou G, Singh B (2002) Nested PCR analysis of Plasmodium parasites. Methods Mol Med 72: 189-203.

19. Duval L, Nerrienet E, Rousset D, Sadeuh Mba SA, Houze S, et al. (2009) Chimpanzee malaria parasites related to Plasmodium ovale in Africa. PLoS ONE 4: e5520. doi:10.1371/journal.pone.0005520.

20. Rubio JM, Benito A, Roche J, Berzosa PJ, García ML, et al. (1999) Semi-nested, multiplex polymerase chain reaction for detection of human malaria parasites and evidence of Plasmodium vivax infection in Equatorial Guinea. Am J Trop Med Hyg 60: 183-187.

21. Ryan JR, Stoute JA, Amon J, Dunton RF, Mtalib R, et al. (2006) Evidence for transmission of Plasmodium vivax among a Duffy antigen negative population in Western Kenya. Am J Trop Med Hyg 75: 575-581.

22. Bedford T, Hartl DL (2008) Overdispersion of the molecular clock: temporal variation of gene-specific substitution rates in Drosophila. Mol Biol Evol 25: 1631-1638.

23. Kumar S, Filipski A, Swarna V, Walker A, Hedges SB (2005) Placing confidence limits on the molecular age of the human-chimpanzee divergence. Proc Natl Acad Sci USA 102: 18842-18847.

24. Drummond AJ, Rambaut A (2003) BEAST version 1.3 (http://evolve.zoo.ox.ac. uk/Evolve/Software.html).

25. Mu J, Joy DA, Duan J, Huang Y, Carlton JM-R, et al. (2005) Host switch leads to emergence of Plasmodium vivax malaria in humans. Mol Biol Evol 22: $1686-1693$. 
26. Hedges SB, Kumar S, editors (2009) The timetree of life. New York: Oxford University Press. pp i-555.

27. Becquet G, Patterson N, Stone AC, Przeworski M, Reich D (2007) Genetic structure of chimpanzee populations. PLoS Genet 3: e66. doi:10.1371/ journal.pgen.0030066.

28. Caswell JL, Mallick S, Richter DJ, Neubauer J, Schirmer C, et al. (2008) Analysis of chimpanzee history based on genome sequence alignments. PLoS Genet 4: e1000057. doi:10.1371/journal.pgen.1000057.

29. Yu N, Jensen-Seaman MI, Chemnick L, Kidd JR, Deinard AS, et al. (2003) Low nucleotide diversity in chimpanzees and bonobos. Genetics 164: 1511-1518.

30. Mobula L, Lilley B, Tshefu AK, Rosenthal PJ (2009) Resistance-mediating polymorphisms in Plasmodium falciparum infections in Kinshasa, Democratic Republic of the Congo. Am J Trop Med Hyg 80: 555-558.

31. Verrelli BC, Tishkoff SA, Stone AC, Touchman JW (2006) Contrasting histories of G6PD molecular evolution and malarial resistance in humans and chimpanzees. Mol Biol Evol 23: 1592-1601.

32. Singh B, Kim Sung L, Matusop A, Radhakrishnan A, Shamsul SSG, et al. (2004) A large focus of naturally acquired Plasmodium knowlesi infections in human beings. Lancet 363: 1017-1024.

33. Cox-Singh J, Davis TME, Lee K-S, Shamsul SSG, Matusop A, et al. (2008) Plasmodium knowlesi malaria in humans is widely distributed and potentially life threatening. Clin Infect Dis 46: 165-171.
34. Tamura K (1992) Estimation of the number of nucleotide substitutions when there are strong transition-transversion and $\mathrm{G}+\mathrm{C}-$ content biases. Mol Biol Evol 9: 678-687.

35. Tamura K, Dudley J, Nei M, Kumar S (2007) MEGA4: Molecular Evolutionary Genetics Analysis (MEGA) software version 4.0. Mol Biol Evol 24: 1596-1599.

36. Yang Z (2007) PAML 4: phylogenetic analysis by maximum likelihood. Mol Biol Evol 24: 1586-15891.

37. Posada D, Crandall KA (2001) Selecting the best-fit model of nucleotide substitution. Syst Biol 50: 580-601.

38. Huelsenbeck JP, Ronquist F (2001) MRBAYES: Bayesian inference of phylogenetic trees. Bioinformatics 17: 754-755

39. Jongwutiwes S, Putaporntip C, Friedman RM, Hughes AL (2002) The extent of nucleotide polymorphism is highly variable across a $3-\mathrm{kb}$ region on Plasmodium falciparum chromosome 2. Mol Biol Evol 19: 1585-1590.

40. Drummond AJ, Ho SY, Phillips MJ, Rambaut A (2006) Relaxed phylogenetics and dating with confidence. PLoS Biol 4: e88. doi:10.1371/journal. pbio.0040088.

41. Rambaut A, Drummond AJ (2003) Tracer version 1.4 (http://beast.bio.ed.ac. uk/Tracer).

42. Bandelt HJ, Forster P, Rohl A (1999) Median-joining networks for inferring intraspecific phylogenies. Mol Biol Evol 16: 37-48. 